\title{
Post-Maxillectomy Orbital Volume Augmentation Using Non- Animal Stabilized Hyaluronic Acid (NASHA) Injection: A Temporary Solution to Complex Reconstruction in Selected Cases
}

\author{
Bijan Beigi* and Deepak Gupta \\ Department of Ophthalmology, Norfolk and Norwich University Hospitals NHS Trust, UK
}

Submission: March 31, 2017; Published: May 04, 2017

*Corresponding author: Bijan Beigi, FRCS, FRCOpth, Department of Ophthalmology, Norfolk and Norwich University Hospitals NHS Trust, Colney Lane, Norwich NR4 7UY, Tel: 01603-288372; Fax: 01603-288261; Email: bb@Mrbeigi.com

\begin{abstract}
Injectable fillers can provide a temporary solution to volume augmentation of the orbit, for functional restoration and improvement in cosmesis; and may be used in sighted globes with caution. They may be a superior option to traditional surgery in selected cases. Minimally invasive approaches have the benefit of reduced operating times and recovery times, can be applied under local anaesthesia, and reduced risk of host and donor site morbidity where non-biological materials are used. In the setting of post-neoplasia reconstruction where the prognosis may be poor, use of minimally invasive, albeit temporary, techniques can help to the improve quality and dignity of life for patients.
\end{abstract}

Keywords: Orbital reconstruction; Fillers

\section{Introduction}

Mid-face surgery involving bony orbital structures can result in significant ophthalmic functional and aesthetic abnormalities requiring reconstruction. We describe a case of post-maxillectomy orbital volume augmentation in a sighted eye using injectable fillers.

\section{Case Report}

A 65-year-old man was referred to the Orbital clinic from the department of Oral Health, following loss of the orbital floor and significant parts of the medial and lateral walls, as a result of maxillectomy for squamous cell carcinoma of the right middle turbinate. There was no ocular or medical history of note. On examination, his visual acuities (VA) were 6/9 right eye and 6/5 left eye. He had a right hypoglobus of $10 \mathrm{~mm}$ without a palate implant, and $6 \mathrm{~mm}$ when in-situ. There was a secondary pseudoupper lid retraction. He had a relative enophthalmos of $8 \mathrm{~mm}$ with the implant in-situ. Ocular movements were full. A HESS chart was recorded (with implant) indicating a right hypoglobus; diplopia was recorded with red/green glasses, suggesting image suppression.

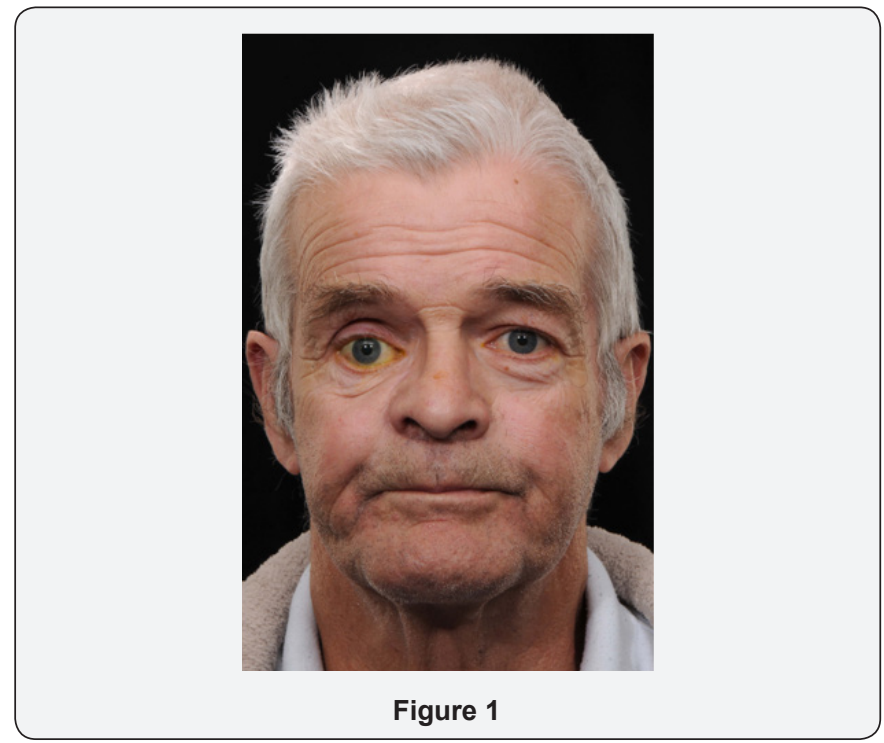

An assessment of his CT scan showed an extensive hemimaxillectomy with resection of the orbital floor, much of the lamina 
papyracea, the maxillary antrum and hard palate. Significant enophthalmos was noted. Soft tissue thickening in the region of the surgical margins was noted (Figure 1). As the patient had undergone post-operative radiotherapy, a conservative approach using NASHA for volume augmentation was decided as an initial step. Floor and medial wall reconstruction with implant/dermo fat graft at the level of the equator of orbit was deferred following discussion with the patient. Correction of lid position would be deferred until later.

He underwent an injection of Orbital (non-animal stabilized hyaluronic acid) NASHA. Retrobulbar block was used comprising of Lignocaine $2 \%$ with adrenaline $1: 200000 ; 1.5 \mathrm{mls}$ was injected, deep, below the globe. The plunger was initially withdrawn to ensure blood vessels were not entered, and a staged augmentation was performed, Three mls of Perlane (Q-Med, Uppsala, Sweden) was injected above the periorbitum of the floor, using an 18G needle. Immediately after the procedure, vision and optic nerve function were monitored and were normal (Compare Figures $2 \&$ 3).
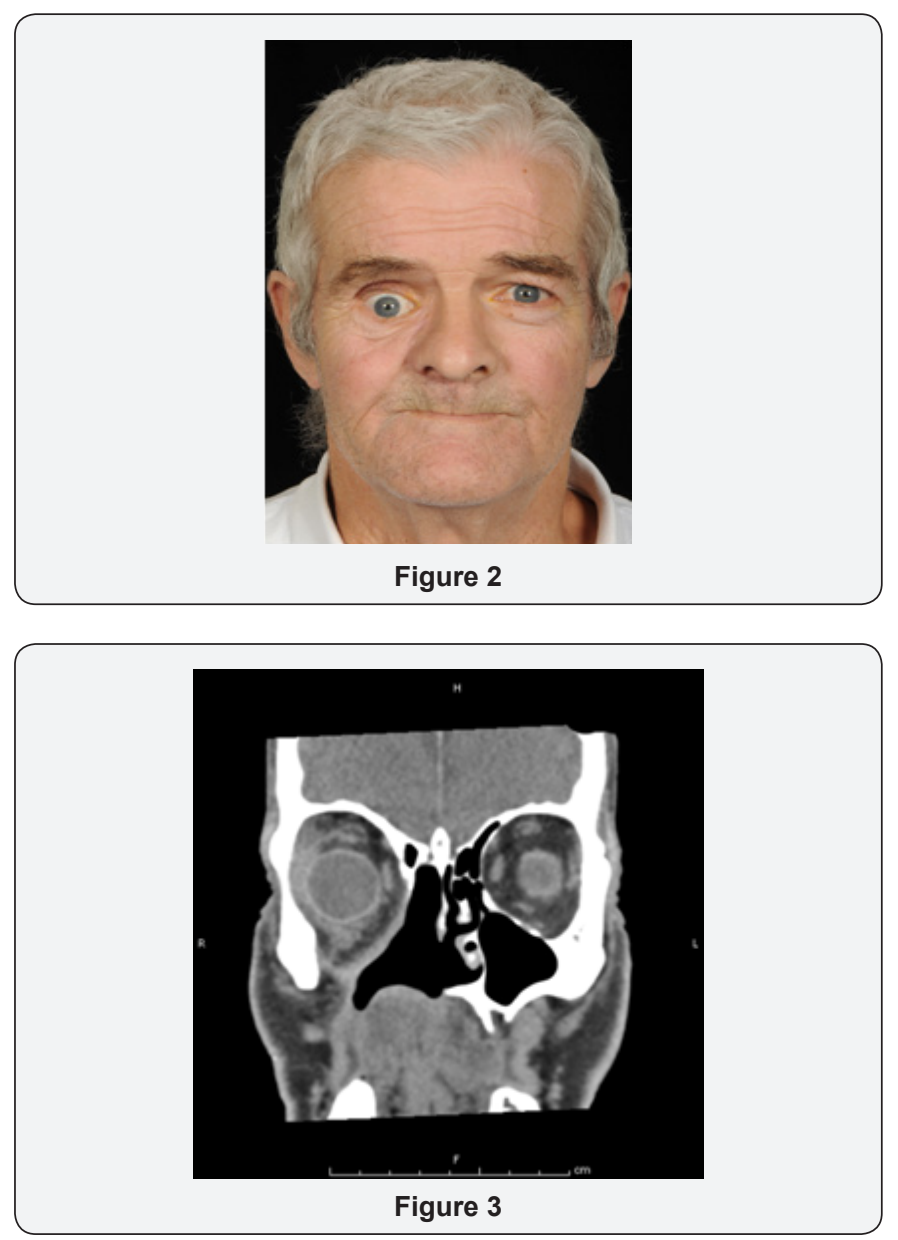

At review 1-week later, a $3 \mathrm{~mm}$ improvement in hyppoglobus and $4 \mathrm{~mm}$ improvement in enophthalmos was noted. However, he was aware of vertical diplopia, and this was controlled with a basedown 3-dioptre prism. This was thought to be due to loss of right eye suppression with the new globe position.
A further review was performed at 8-months post-injection and a slight increase in enophthalmos and hypoglobus were noted.At 13-months after the initial injection, encouraged by initial results, a further injection of $3 \mathrm{mls}$ of Perlane (Q-Med, Uppsala, Sweden) was injected using the same technique as before with tightening of the lower lid by means of a lateral canthal elevation. This resulted in an improvement in hypoglobus to $2-3 \mathrm{~mm}$. His diplopia persisted without prisms, although over the next 8-months started coping better without prisms and the diplopia resolved with normal binocular single vision. The long-term plan was to continue with annual orbital NASHA injections.

\section{Discussion}

From an ophthalmic point of view, mid-face reconstruction aims to provide support to orbital contents, minimize changes in globe position, orbital volume and eyelid function [1]. Symmetrical orbital volume is a requirement for binocular single vision. Complications following reconstructive surgery, and radiotherapy, include resorption of orbital fat and wound contracture, exacerbating enophthalmos and diplopia. Post-maxillectomy radiation therapy increases the risk of orbital sequelae, such as fat atrophy and scar formation, and ocular sequelae, such as optic atrophy, cataract formation, ocular surface dryness, and ectropion [2].

Orbital reconstruction may range from minimal to complex. Minimal bone resections or small orbital floor defects may not require reconstruction. On the other end of the spectrum, subtotal/ total floor defects ( $>75 \%$ surface area) and multi segmental defects will necessitate rigid reconstruction. Primary reconstruction of total maxillectomy defects with preservation of orbital contents remains a complex problem without a perfect solution [3]. In a case series of 66 patients with sinonasal malignancy that had eye-sparing surgery, [2] the most common abnormality following subsequent reconstruction was globe mal position (enophthalmos or hypophthalmos) (34/66 patients, 63\%), associated with lack of adequate rigid reconstruction of subtotal or total orbital floor or multisegment orbital defects [2]. The problem can be exacerbated with post-operative radiotherapy [4].

In our case, the patient was keen for correction of his vertical dystopia from a functional and cosmetic point of view. Surgery would have been quite complex due to the absence of the floor, medial and lateral walls. The option of volume augmenatation with a dermo fat graft was considered, but there was a possibility that this graft might not survive due to lack of circulation. There was also a risk of recurrence of the original pathology. A conservative approach was taken and floor augmentation with NASHA was decided as the first stage of the procedure. This addressed both the hypoglobus and enophthalmos to the patient's satisfaction, to the extent that need for further orbital surgery was obviated. Furthermore, binocular single vision returned with the use of prisms.

There are no reports addressing reconstruction of the orbital component of the post-maxillectomy defect using injectable 
materials for volume augmentation that we are aware of. A minimally invasive approach to orbital volume augmentation using agents such as injectable calcium hydroxylapatite (CaHA), polyacrylamide gel, hydrogel pellet expanders, micro-fat grafts and NASHA have been well described in the anophthalmic socket (post-enucleation socket syndrome). There are only a handful of reports in the context of sighted globes. These include silent sinus syndrome, [5] deep set eyes, [6,7] trauma, [7] and facial hemiatrophy (Parry Romberg syndrome) [7]. We are not aware of any reports of use of filler in the context of carcinoma.

NASHA is produced from non-animal sources by bacterial fermentation. It is well tolerated, has a low immunogenic potential, and there is low risk of antigenic contamination and hypersensitivity reactions [8]. A major advantage in sighted globes is that its effects can be reversed by dissolving it with hyaluronidase. Disadvantages include a relatively short life span compared to other products and the requirement to inject through an 18G needle in order to maintain particle integrity [8]. However, blunt cannulas can be used. Some studies have reported a 1:1 correlation between the desired augmentation volume (enophthalmos reduction) and each millimetre of product injected, [7] whilst others have not [9]. (We feel the augmentation effect depends on the remaining bony walls and the integrity of the periorbita). Twelve months is the longest duration of action reported [7,9]. Injection of NASHA is recommended in the anterior orbit, and behind the equator of the globe, in order to achieve globe elevation and axial globe advancement respectively $[7,9,10]$. Injections may be intraconal or extraconal. Proponents of the former cite better globe advancement and reduced anterior migration of filler; [11] proponents of the latter cite risk of vascular compression in sighted globes with intraconal placement [8].

Possible complications include risk of intravascular injection and embolization with loss of vision in sighted eyes. Commonly reported complications include injection site tenderness, ecchymosis and chemosis, [12] stimulation of oculocardiac reflex and associated hypotension, [7,8,12] transient ptosis, [6] anterior migration of filler [13] and inflammatory orbital cellulitis [9].

The benefits of a minimally invasive approach over traditional surgery include reduced operating times, application under local anaesthesia, reduced recovery time and reduced risk of host and donor site morbidity where non-biological materials are used. The benefits need to be weighed against the risks. In the setting of post-neoplasia reconstruction where the prognosis may be poor, use of minimally invasive, albeit temporary, techniques can help to the improve quality and dignity of life for patients, especially where resorting to more complex surgical procedures may be too demanding for the patient in view of their morbidity and reduced life-expectancy [14].

In summary, injectable fillers can provide a temporary solution to volume augmentation of the orbit, for functional restoration and improvement in cosmesis; and may be used in sighted globes with caution. They may be a superior option to traditional surgery in selected cases.

\section{References}

1. Andrades P, Militsakh O, Hanasono MM, Rieger J, Rosentha EL (2011) Current Strategies in Reconstruction of Maxillectomy Defects. Arch Otolaryngol Head Neck Surg 137(8): 806-812.

2. Imola MJ, Schramm VL (2002) Orbital preservation in surgical management of sinonasal malignancy. Laryngoscope 112(8 Pt 1): $1357-1365$.

3. Lekawale H, Chitharanjan A (2012) Total maxillectomy with orbital clearance for squamous cell carcinoma of maxilla- our experience with two cases and literature review. Indian J Appl Res 2(3): 149-152.

4. Suárez C, Ferlito A, Lund VJ, Silver CE, Fagan JJ, et al. (2008)Management of the orbit in malignant sinonasal tumors. Head Neck 30(2): 242-250.

5. Mavrikakis I, Detorakis ET, Yiotakis I, Kandiloros D (2012) Non surgical management of silent sinus syndrome with hyaluronic acid gel. Ophthal Plast Reconstr Surg 28(1): e6-e7.

6. Malhotra R (2007) Deep orbital Sub-Q restylane (non animal stabilized hyaluronic acid) for orbital volume enhancement in sighted and anophthalmic orbits. Arch Ophthalmol 125(12): 1623-1629.

7. Hardy TG, Joshi N, Kelly MH (2007) Orbital volume augmentation with autologous micro-fat grafts. Ophthal Plast Reconstr Surg 23(6): 445449 .

8. Vagefi MR (2013) Minimally invasive approaches to orbital volume augmentation. Int Ophthalmol Clin 53(3): 67-86.

9. Zamani M, Thyagarajan S, Olver JM (2010) Adjunctive use of hyaluronic acid gel (Restylane Sub-Q) in anophthalmic volume deficient sockets and phthisical eyes. Ophthal Plast Reconstr Surg 26(4): 250-253.

10. Vagefi MR, McMullan TF, Burroughs JR, White GL Jr, McCann JD, et al. (2007)Injectable calcium hydroxylapatite for orbital volume augmentation. Arch Facial Plast Surg 9(6): 439-442.

11. Tay E, Olver J (2008) Intraorbital hyaluronic acid for enophthalmos. Ophthalmology 115(6): 1101-1101.

12. da Silva AL, Bredemeier M, Gebrim ES, Moura Eda M (2008)Intra orbital polyacrylamide gel injection for the treatment of anophthalmic enophthalmos. Ophthal Plast Reconstr Surg 24(5): 367-371.

13. Buchanan AG, Holds JB, Vagefi MR, Bidar M, McCann JD, et al. (2012) Anterior filler displacement following injection of calcium hydroxylapatite gel (Radiesse) for anophthalmic orbital volume augmentation. Ophthal Plast Reconstr Surg 28(5): 335-337.

14. Vagefi MR, Mc Mullan TF, Burroughs JR, Georgescu D, Mc Cann JD, et al. (2011) Orbital augmentation with injectable calcium hydroxylapatite for correction of postenucleation/evisceration socket syndrome. Ophthal Plast Reconstr Surg 27(2): 90-94. 
This work is licensed under Creative Commons Attribution 4.0 License DOI: $10.19080 / J 0 J 0.2017 .03 .555607$
Your next submission with Juniper Publishers will reach you the below assets

- Quality Editorial service

- Swift Peer Review

- Reprints availability

- E-prints Service

- Manuscript Podcast for convenient understanding

- Global attainment for your research

- Manuscript accessibility in different formats

(Pdf, E-pub, Full Text, Audio)

- Unceasing customer service

Track the below URL for one-step submission https://juniperpublishers.com/online-submission.php 